\title{
VALORACIÓN DE LA CALIDAD DEL SERVICIO TURÍSTICO DE CARTAGENA DE INDIAS, COLOMBIA
}

Juan Carlos Vergara Schmalbach Universidad de Cartagena Colombia

María Carolina Cortez Cantero Universidad de Cartagena Colombia

María Fernanda Martínez Correa Universidad de Cartagena Colombia 
Panorama Económico, Vol. 27 - No. 2 (Abril - Junio de 2019), pp. 544-558

Juan Carlos Vergara Schmalbach $\mid$ María Carolina Cortez Cantero $\mid$ María Fernanda Martínez Correa JEL: L80, L82, L88

\title{
Valoración de la calidad del servicio turístico de Cartagena de Indias, Colombia Resumen
}

Cartagena de Indias (Colombia) se ha mantenido como la tercera ciudad del país donde convergen la mayor proporción de turistas nacionales, presentando, además, un creciente aumento en las llegadas de turistas internacionales. Para continuar con esta ruta de crecimiento, el gobierno local y empresarios deben concentrar sus esfuerzos en vigilar e incrementar la satisfacción de los turistas, con el fin de atraer nuevos visitantes y fidelizar a aquellos que ya han visitado la ciudad. En este sentido, el presente trabajo tiene como objetivo mostrar los resultados de la valoración de la calidad del servicio en la ciudad, a partir de los productos y servicios ofrecidos y la identificación de sus atributos, con el fin de diseñar recomendaciones y estrategias que apunten al mejoramiento del servicio en el destino, su promoción e imagen. Lo anterior se logró través de la determinación de los factores clave de su estadía e intenciones de nuevas visitas mediante la aplicación de un modelo de ecuaciones estructurales, estableciendo relaciones significativas entre atributos e identificando los elementos clave que afectan la satisfacción. Para llevar a cabo el estudio, se aplicaron 384 encuestas definitivas a turistas nacionales, durante la temporada de fin de año (2017-2018) que permitieron la validación del modelo propuesto, con condiciones generales que facilitarán la evaluación del servicio turístico de Cartagena. Se concluye que las dimensiones como empatía, capacidad de respuesta y tangibilidad tienen influencia en la satisfacción. A su vez, la satisfacción resultó ser un aspecto determinante de las intenciones futuras del turista nacional.

Palabras clave: Evolución, Validación, Selección de modelos, Economía Regional, Estudios industriales, Turismo, Desarrollo

\section{Évaluation de la qualité du service touristique à Carthagène des Indes, Colombie \\ Résumé}

Carthagène des Indes (Colombie) est restée la troisième ville du pays où converge la plus grande proportion de touristes nationaux, présentant également une augmentation croissante des arrivées de touristes internationaux. Pour continuer sur cette voie de croissance, le gouvernement local et les hommes d'affaires doivent concentrer leurs efforts sur le suivi et l'augmentation de la satisfaction des touristes, afin d'attirer de nouveaux visiteurs et de fidéliser ceux qui ont déjà visité la ville. En ce sens, le présent travail vise à montrer les résultats de l'évaluation de la qualité de service dans la ville, sur la base des produits et services offerts et l'identification de leurs attributs, afin de concevoir des recommandations et des stratégies qui pointent vers l'amélioration de la service dans la destination, sa promotion et son image. Cela a été réalisé grâce à la détermination des facteurs clés de votre séjour et à l'intention de nouvelles visites en appliquant un modèle d'équation structurelle, en établissant des relations significatives entre les attributs et en identifiant les éléments clés qui affectent la satisfaction. Pour mener à bien l'étude, 384 enquêtes définitives ont été appliquées aux touristes nationaux au cours de la saison de fin d'année (2017-2018) qui ont permis la validation du modèle proposé, avec des conditions générales qui faciliteront l'évaluation du service touristique de Carthagène. Il est conclu que les dimensions telles que l'empathie, la réactivité et la tangibilité ont une influence sur la satisfaction. À son tour, la satisfaction s'est avérée être un aspect déterminant des intentions futures du tourisme national.

Mots clés: Évolution, validation, sélection de modèles, économie régionale, études industrielles, tourisme, développement

\begin{abstract}
Assessment of the quality of the tourist service in Cartagena de Indias, Colombia Abstract

Cartagena de Indias (Colombia) has remained the third city in the country where the largest proportion of national tourists converge, also presenting a growing increase in international tourist arrivals. To continue on this growth path, the local government and businessmen must concentrate their efforts on monitoring and increasing the satisfaction of tourists, in order to attract new visitors and retain those who have already visited the city. In this sense, the present work aims to show the results of the assessment of service quality in the city, based on the products and services offered and the identification of their attributes, in order to design recommendations and strategies that point to the improvement of the service in the destination, its promotion and image. This was achieved through the determination of the key factors of your stay and the intention of new visits by applying a structural equation model, establishing significant relationships between attributes and identifying the key elements that affect satisfaction. To carry out the study, 384 definitive surveys were applied to national tourists during the year-end season (2017-2018) that allowed the validation of the proposed model, with general conditions that will facilitate the evaluation of the Cartagena tourist service. It is concluded that the dimensions such as empathy, responsiveness and tangibility have an influence on satisfaction. In turn, satisfaction turned out to be a determining aspect of the future intentions of the national tourist.
\end{abstract}

Keywords: Evolution, Validation, Selection of models, Regional Economics, Industrial studies, Tourism, Development 


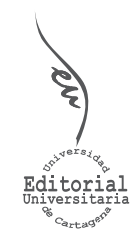

Artículo de Investigación

\section{Valoración de la calidad del servicio turístico de Cartagena de Indias, Colombia'}

INFORMACIÓN DEL ARTícuLO

Recepción del artículo: 05/01/2019

Concepto de evaluación: 16/02/2019

Aceptación del artículo: 21/03/2019
Juan Carlos Vergara Schmalbach ${ }^{2}$

Universidad de Cartagena, Colombia

María Carolina Cortez Cantero Universidad de Cartagena, Colombia

María Fernanda Martínez Correa Universidad de Cartagena, Colombia

\section{INTRODUCCIÓN}

Cartagena de Indias ha sido desde siempre una ciudad excepcional. Considerada como uno de los puertos más estratégicos de la corona española. En la época de la colonia, la ciudad se mantuvo como un importante eje comercial, soportando ataques y agravios durante el período independentista. En la actualidad, Cartagena de Indias se constituye como la ciudad con mayor vocación turística del país por sus atractivos históricos, culturales y ambientales con una ubicación privilegiada en el Caribe.

En el año 2018, Cartagena de Indias alcanzó la primera posición en el Índice de Competitividad Turística Regional, entre todas las ciudades con vocación turística de Colombia, calificada por tener un desempeño sobresaliente en aspectos económicos, empresariales y sociales (Centro de Pensamiento Turístico - Colombia, 2018). En cuanto al número de turistas nacionales en el año 2018, la ciudad se ubicó en el tercer puesto en el país donde convergen la mayor proporción de turistas nacionales y la segunda en cuanto a la visita de extranjeros (Redacción Economía, 2019), manteniendo, en ambos casos, una tasa de crecimiento anual en la llegada de turistas. Con respecto a la llegada de cruceros, la ciudad mantiene el primer puesto desde el año 2000, registrando 217 cruceros en el año 2018 (Centro de Información Turística de Colombia, 2018). 
Para continuar con esta ruta de crecimiento, el gobierno local y empresarios deberán concentrar sus esfuerzos en vigilar e incrementar la satisfacción de los turistas, con el fin de atraer nuevos visitantes y fidelizar a aquellos que ya han visitado la ciudad. En este sentido, el presente artículo tiene como objetivo proponer un esquema o modelo para valorar la calidad del servicio en Cartagena de Indias, a partir de los productos y servicios turísticos ofrecidos y la identificación de sus atributos, con el fin de diseñar recomendaciones y estrategias que le apunten al mejoramiento del servicio en el destino, su promoción e imagen. Lo anterior se logró a través de la determinación de los factores clave de su estadía e intenciones de nuevas visitas mediante la aplicación de un modelo de ecuaciones estructurales a través de la técnica Mínimos Cuadrados Parciales (o PLS, por sus siglas en inglés), que permitieron analizar el servicio turístico de Cartagena de Indias, estableciendo las relaciones significativas entre atributos e identificando los elementos clave que afectan la satisfacción del turista nacional.

\section{LA CALIDAD DEL SERVICIO TURÍSTICO}

El estudio de la calidad de servicio es fundamental para su aplicación en la medición del grado de satisfacción de los turistas. La calidad de servicio se identifica como una construcción de naturaleza compleja (Gronroos, 1982), difusa y abstracta (Zeithaml, 1981) y se asocia a tres condiciones únicas e inherentes a los servicios. Estas condiciones se pueden resumir en la intangibilidad, la heterogeneidad y la inseparabilidad de la producción y del consumo (Zeithaml, Berry, \& Parasuraman, 1988).

Otros elementos como la calidad objetiva y subjetiva, amplían aún más los enfoques para valorar la calidad del servicio, desde el punto de vista funcional o desde las percepciones de los usuarios del mismo (DA Garvin, 1987; Zeithaml et al., 1988). Holbrook (2002) distingue la calidad mecánica (objetiva) de la calidad de la humanística (subjetiva), argumentando que la mecánica tiene que ver con las características objetivas de una cosa o evento y la humanística, hace mención al conjunto de respuestas subjetivas de las personas ante los objetos, independizando los abordajes hacia la valoración de la calidad.

La calidad objetiva propone los primeros acercamientos del análisis de la calidad de un servicio o producto. Esta se puede definir como el nivel de conformidad con relación a las especificaciones definidas por las marcas (Crosby, 1992). Este fenómeno se asemeja a una visión funcional, en la cual se deben controlar todas las etapas que intervienen en la prestación de un servicio, el cuál pudiese ser comparada con la fabricación de cualquier bien (Garvin, 1984). En el enfoque de calidad subjetiva el cliente o usuario tiene una mayor preponderancia, puesto que es él quien percibe construye una visión de la calidad del servicio recibido. De acuerdo con los postulados de Zeithaml, Parasuraman, \& Berry (1990), la calidad es el resultado de una comparación entre las expectativas y sus percepciones del desempeño de las empresas o marcas que prestan el servicio.

Para estos autores la calidad es una forma de actitud, que se relaciona con la satisfacción, pero no se ve definida estrictamente ésta (Zeithaml et al., 1988). Según este postulado, la calidad que se percibe de una empresa va ligada a la percepción del cliente acerca de cómo el servicio satisface o no sus expectativas (VergaraSchmalbach, Quesada, \& Blanco, 2012). Por ello la subjetividad le otorga a la calidad un carácter cambiante y relativo, sujeto al tiempo y el espacio (Holbrook, 2002). Es 
así como calidad de servicio, se refiere a un concepto multidimensional que está estrechamente ligado a las expectativas y percepciones de los usuarios del servicio.

\section{Modelo conceptual propuesto}

A partir del modelo original planteado sobre la calidad percibida del servicio de Cronin y Taylor (1992) y adaptado a los estudios señalados anteriormente, se propuso el siguiente esquema para la aplicación del modelo teórico el cual permite identificar la relación que existe entre la percepción, la satisfacción y las intenciones futuras, donde se agruparon los diferentes factores que van a hacer evaluados, conforme a la percepción, satisfacción e intenciones futuras, para lograr valoración de la calidad del servicio del sector turístico de la ciudad de Cartagena de Indias (Gráfico 1).

Gráfica 1. Modelo propuesto para el planteamiento de las hipótesis para medir el grado de satisfacción de los turistas nacionales en la ciudad de Cartagena de Indias.

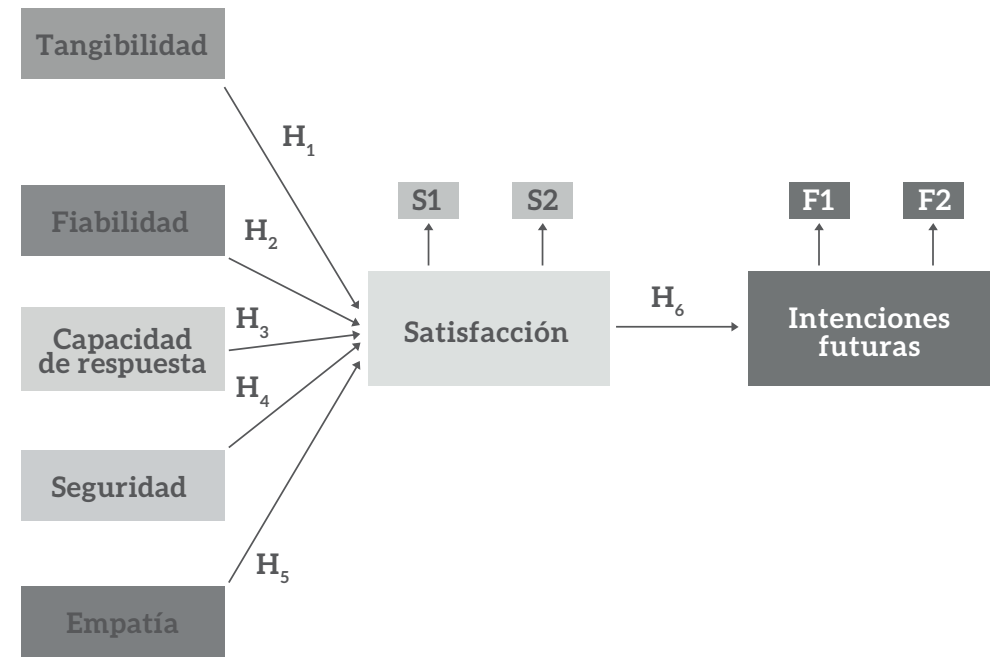

Fuente: Elaborado por los autores

En el modelo se identificaron seis (6) hipótesis fundamentadas en posibles relaciones causales entre las variables latentes o estructurales presentes, con el objetivo de evaluar el nivel de correlación que existe entre las dimensiones de calidad utilizadas en el estudio y su efecto directo sobre la satisfacción del turista nacional. Se denota como $\mathrm{HO}$ a las hipótesis nulas y $\mathrm{H} 1$ a las hipótesis alternativas.

$\mathrm{H}_{\mathrm{o}}$ : Existe un efecto causal directo entre las variables de tangibilidad y la satisfacción del turista nacional.

$\mathrm{H}_{1}$ : No existe un efecto causal directo entre las variables de tangibilidad y la satisfacción del turista nacional.

$\mathrm{H} 2_{0}$ : Existe un efecto causal directo entre las variables de fiabilidad y la satisfacción del turista nacional. 
$\mathrm{H} 2_{1}$ : No existe un efecto causal directo entre las variables de fiabilidad y la satisfacción del turista nacional.

H3: Existe un efecto causal directo entre las variables de capacidad de respuesta y la satisfacción del turista nacional.

H3: No existe un efecto causal directo entre las variables de capacidad de respuesta y la satisfacción del turista nacional.

$\mathrm{H}_{0}$ : Existe un efecto causal directo entre las variables de seguridad y la satisfacción del turista nacional.

$\mathrm{H}_{1}$ : No existe un efecto causal directo entre las variables de seguridad y la satisfacción del turista nacional.

H5: Existe un efecto causal directo entre las variables de empatía y la satisfacción del turista nacional.

H5: No existe un efecto causal directo entre las variables de empatía y la satisfacción del turista nacional.

H6: Existe un efecto causal directo entre las variables de satisfacción e intenciones futuras del turista nacional.

H6: No existe un efecto causal directo entre las variables de satisfacción e intenciones futuras del turista nacional.

De acuerdo con las características de la ciudad y considerando las variables de la calidad del servicio se propusieron una serie de variables para valorar las percepciones de acuerdo con las 5 dimensiones esquematizadas en el modelo (tangibilidad, fiabilidad, capacidad de respuestas, empatía y seguridad), descritas en la Tabla 1.

Tabla 1.

Ítems valorados por dimensión del servicio

\begin{tabular}{cl}
\hline Código & \\
\hline TANGIBILIDAD & \\
\hline P1 & Preguntas \\
P2 & Estado de la infraestructura (andenes, calles y edificaciones) \\
P3 & Preservación de los atractivos y monumentos turísticos \\
P4 & Calidad de las instalaciones \\
P5 & Limpieza del lugar donde se hospedó \\
P6 & Variedad y cantidad de restaurantes de la ciudad \\
P7 & Calidad de las instalaciones de los restaurantes \\
P8 & Limpieza de los restaurantes \\
P9 & Limpieza de las playas \\
P10 & Infraestructura turística en las zonas de playa \\
P11 & Limpieza de la ciudad
\end{tabular}

FIABILIDAD 
Tabla 1.

Ítems valorados por dimensión del servicio

\begin{tabular}{|c|c|}
\hline Código & Preguntas \\
\hline \multicolumn{2}{|c|}{ FIABILIDAD } \\
\hline P15 & Relación calidad-precio de los servicios \\
\hline P16 & Tarifa cobrada en los recorridos por los taxistas \\
\hline P17 & Facilidad de movilidad peatonal \\
\hline P18 & Movilidad y transporte en la ciudad \\
\hline \multicolumn{2}{|c|}{ CAPACIDAD DE RESPUESTA } \\
\hline P19 & Accesibilidad a los atractivos turísticos \\
\hline P20 & $\begin{array}{l}\text { Accesibilidad de los atractivos turísticos para las personas con } \\
\text { movilidad reducida }\end{array}$ \\
\hline P21 & Conocimiento de los guías respecto a la historia de la ciudad \\
\hline P22 & Accesibilidad de la información acerca de los atractivos turísticos \\
\hline \multicolumn{2}{|c|}{ SEGURIDAD } \\
\hline P23 & Seguridad en su recorrido por el centro histórico \\
\hline P24 & Seguridad en su estancia en la playa \\
\hline P25 & Seguridad que se percibe en la ciudad \\
\hline \multicolumn{2}{|l|}{ EMPATÍA } \\
\hline P26 & Cordialidad y respeto de los vendedores informales \\
\hline P27 & Atención prestada por el personal de los atractivos turísticos \\
\hline P28 & Amabilidad, buen trato de los guías \\
\hline P29 & Presentación personal de los guías \\
\hline P30 & Calidad de la atención al cliente en el lugar que lo hospedó \\
\hline P31 & Calidad de la atención al cliente en los restaurantes \\
\hline P32 & Respeto y cordialidad de los vendedores ambulantes \\
\hline P33 & Experiencia al visitar las playas \\
\hline P34 & Hospitalidad del cartagenero \\
\hline P35 & Vocación de servicio de los cartageneros en general \\
\hline P36 & Trato de los taxistas \\
\hline
\end{tabular}

Fuente: Elaborado por los autores

Además de las preguntas anteriores, se incluyeron dos preguntas relacionadas con el tema de satisfacción general por el servicio recibido y dos preguntas relacionadas con las intenciones futuras de los turistas (recomendación a terceros e intención de regresar a la ciudad).

\section{MARCO METODOLÓGICO}

Para la interpretación de los datos del modelo del presente estudio, se aplicó el método de ecuaciones estructurales (Structural Equation Modeling, SEM) y la técnica de Mínimos Cuadrados Parciales que combina tanto la regresión múltiple 
como el análisis factorial. Este método permite no solo evaluar las interrelaciones de dependencia, sino también, incorporar los efectos del error de medida sobre los coeficientes estructurales al mismo tiempo (Hair, J.F.; Anderson, R.E.; Tatham, R.L. \& Black, 1999).

\section{Materiales y método}

Para obtener información relevante de la investigación, se diseñó un instrumento (tipo encuesta) estructurado, basado en una escala Likert de 5 puntos por cada uno de los ítems que evaluaron las percepciones de los turistas. Esta forma de capturar los datos de los usuarios es la más utilizada en los métodos de medición para la valoración de la calidad de servicios (Vergara-Schmalbach \& Quesada, 2013). Para la percepción de atributos, productos y servicios de la ciudad, se estableció una escala de puntación con valores de 0 a 5, Siendo 0 = No conoce; 1 = Calificación mínima y $5=$ Calificación máxima.

Para la satisfacción de la experiencia, se planteó una escala Likert de 1 a 5 puntos, ajustada a la información que se desea recopilar de los encuestados, tales como:

- Una escala de 1 a 5, que refleje el grado de satisfacción que se percibe de los servicios recibidos durante la estancia en la ciudad de Cartagena de Indias, siendo 1= Mucho peor a lo esperado y 5= Mucho mejor de lo esperado.

- Una escala de 1 a 5, que exprese el nivel de satisfacción general con respecto a su estancia en la ciudad de Cartagena de Indias, siendo 1= Muy insatisfecho y $5=$ Muy satisfecho.

Por último, para medir las intenciones futuras, se planteó la misma escala de medición utilizada en la satisfacción, que va desde 1 a 5 puntos, refiriéndose a que tan dispuesto estaría el encuestado de recomendar a un familiar, amigo o compañero, el visitar Cartagena de Indias, siendo 1= Poco dispuesto y 5= Muy dispuesto.

\section{Muestra del estudio}

Para el desarrollo del proyecto se aplicaron 392 encuestas a turistas nacionales, durante la temporada de fin de año, que va desde diciembre de 2017 a enero de 2018. Durante el análisis, solo fueron aceptados 384 registros, dejando por fuera 8 registros considerados como inconsistentes (con datos faltantes y valores que no se ajustaron a la escala de medición). La aplicación del instrumento se realizó a través de 14 encuestadores, los cuales estuvieron ubicados en los sitios de interés turísticos de la ciudad, en horarios de mayor afluencia turística.

\section{RESULTADOS}

De los 384 registros válidos, los resultados arrojaron que el 55\% de los encuestados manifestaron pertenecer al género femenino. Las edades de los encuestados se concentraron en un rango entre los 18 y los 47 años, con un promedio de 27 años. El 45\% indicó ser soltero, seguido por un 32\% que aseguró estar casado y un 19\% que se encuentra en unión libre. Una minoría compuesta por un 3\% mencionó estar separado. En cuanto a las preguntas orientadas a evaluar la calidad del servicio, en la Tabla 2 se muestra un resumen de los principales indicadores estadísticos. 
Tabla 2.

Resultados estadísticos por variable

\begin{tabular}{|c|c|c|c|c|}
\hline Variable & Media & Mediana & $\begin{array}{l}\text { Desviación } \\
\text { Estándar }\end{array}$ & Kurtosis \\
\hline P1 & 3.818 & 4.000 & 0.938 & 2.045 \\
\hline P2 & 3.760 & 4.000 & 1.106 & 0.247 \\
\hline P3 & 4.059 & 4.000 & 1.071 & 4.872 \\
\hline P4 & 3.094 & 4.000 & 2.025 & -1.243 \\
\hline P5 & 3.227 & 4.000 & 2.089 & -1.229 \\
\hline P6 & 4.041 & 4.000 & 1.386 & 2.927 \\
\hline P7 & 3.770 & 4.000 & 1.501 & 1.635 \\
\hline P8 & 3.749 & 4.000 & 1.518 & 1.466 \\
\hline P9 & 3.327 & 4.000 & 1.350 & 0.499 \\
\hline P10 & 3.379 & 4.000 & 1.357 & 0.678 \\
\hline P11 & 3.510 & 4.000 & 1.059 & 0.680 \\
\hline P12 & 2.602 & 3.000 & 1.450 & -0.806 \\
\hline P13 & 3.026 & 4.000 & 2.024 & -1.308 \\
\hline P14 & 3.100 & 3.000 & 1.537 & -0.361 \\
\hline P15 & 2.752 & 3.000 & 1.424 & -0.601 \\
\hline P16 & 2.870 & 3.000 & 1.626 & -0.938 \\
\hline P17 & 3.540 & 4.000 & 1.302 & -0.214 \\
\hline P18 & 4.066 & 4.000 & 1.008 & 1.860 \\
\hline P19 & 3.890 & 4.000 & 1.286 & 2.790 \\
\hline P20 & 2.031 & 2.000 & 1.807 & -1.472 \\
\hline P21 & 2.673 & 4.000 & 2.188 & -1.735 \\
\hline P22 & 3.406 & 4.000 & 1.621 & -0.104 \\
\hline P23 & 3.679 & 4.000 & 1.108 & 0.783 \\
\hline P24 & 4.306 & 5.000 & 1.034 & 5.433 \\
\hline P25 & 3.492 & 4.000 & 1.488 & 0.207 \\
\hline P26 & 3.528 & 4.000 & 1.169 & 0.673 \\
\hline P27 & 3.469 & 4.000 & 1.650 & 0.171 \\
\hline P28 & 2.798 & 4.000 & 2.208 & -1.704 \\
\hline P29 & 2.712 & 4.000 & 2.131 & -1.667 \\
\hline P30 & 3.199 & 4.000 & 2.066 & -1.210 \\
\hline P31 & 3.673 & 4.000 & 1.586 & 0.843 \\
\hline P32 & 3.072 & 3.000 & 1.452 & -0.537 \\
\hline P33 & 3.872 & 4.000 & 1.375 & 2.112 \\
\hline P34 & 4.125 & 4.000 & 0.935 & 1.607 \\
\hline P35 & 3.995 & 4.000 & 1.028 & 2.717 \\
\hline P36 & 3.293 & 4.000 & 1.661 & -0.468 \\
\hline S1 & 4.122 & 4.000 & 0.729 & 0.432 \\
\hline S2 & 4.296 & 4.000 & 0.658 & -0.486 \\
\hline IF1 & 4.528 & 5.000 & 0.703 & 7.215 \\
\hline IF2 & 4.594 & 5.000 & 0.757 & 9.096 \\
\hline
\end{tabular}

Fuente: Elaborado por los autores

Para la solución del modelo propuesto se empleó la técnica SEM-PLS (basado en varianzas), técnica que puede ser empleada para el desarrollo de proyectos explicativos y predictivos (F. Hair Jr, Sarstedt, Hopkins, \& G. Kuppelwieser, 2014) con muestras pequeñas, cuyos datos no se basan en un comportamiento normal (Cassel, Hackl, \& 
Westlund, 1999; Chin \& Newsted, 1999; Jorg Henseler, Ringle, \& Sinkovics, 2009). La solución del modelo se sustentó en el esquema de relaciones ponderadas, esquema que provee más altos valores para R2 para las variables latentes endógenas (Monecke \& Leisch, 2012), ajustadas a las investigaciones de tipo explicativas, que tienen por objetivo establecer relaciones entre un fenómeno y la teoría que la sustenta. El esquema conceptual o teórico se construyó teniendo en cuenta un modelo formativo, donde las variables observables afectan las variables latentes o estructurales (F. Hair Jr et al., 2014). Este modelo permitió explicar los constructos o dimensiones desde los indicadores evaluados en el instrumento (Gráfico 2).

\section{Gráfica 2. Modelo estructural resuelto mediante la técnica PLS}

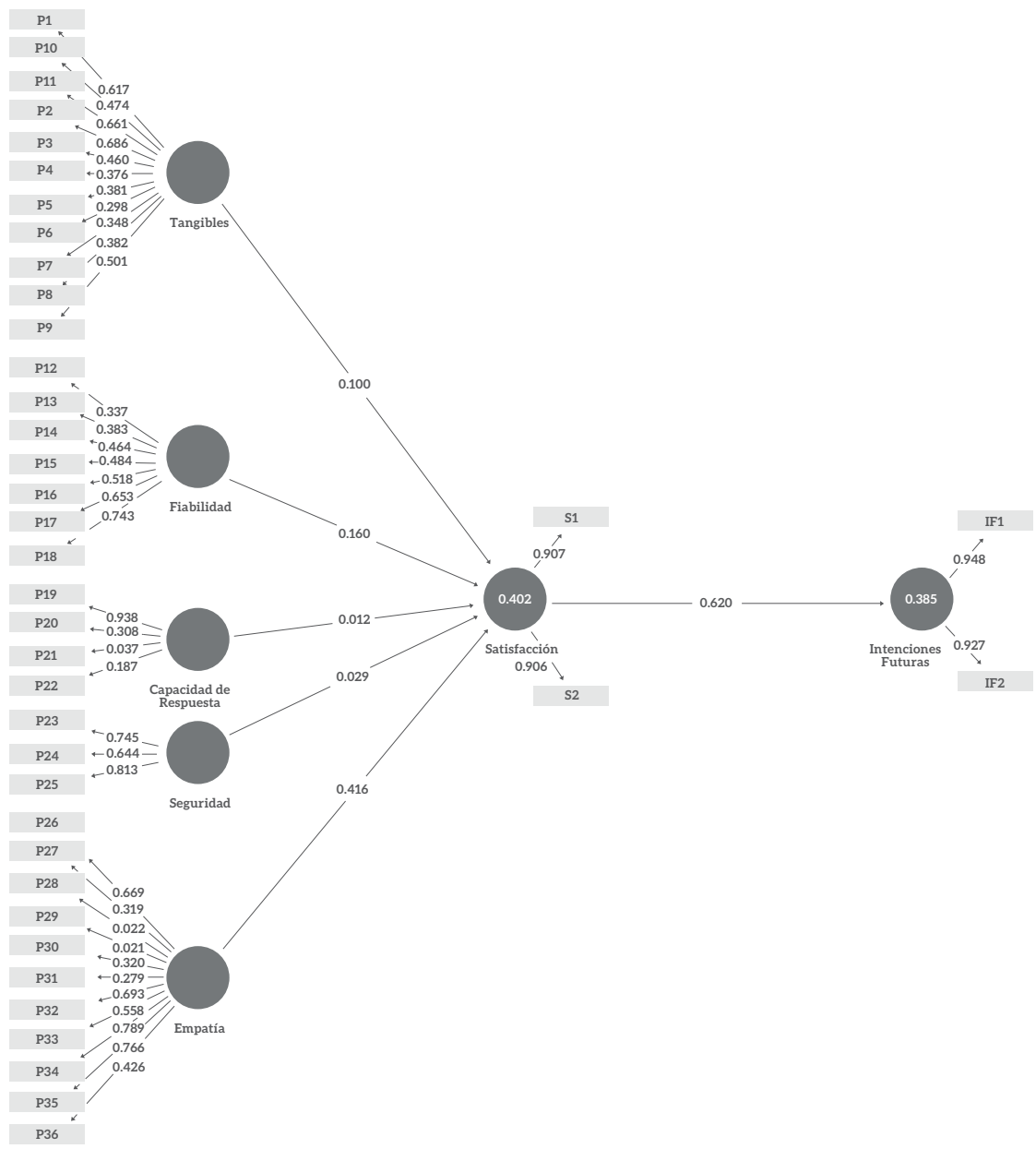

Fuente: Elaborado por los autores

Las correlaciones entre capacidad de respuesta y satisfacción (0.012), y seguridad y satisfacción $(0,029)$ estuvieron cercanas a cero. Esto significa que en dichas variables no se evidenció una correlación significativa (para un $\mathrm{p}<=0,05$ ). Basados en el valor de 
R2 (varianza explicada) se tiene que el modelo explica en un 40.2\% la variación de la variable Satisfacción y en un 38.5, la variación de la variable intenciones futuras.

De acuerdo con el modelo inicial presentado, y teniendo en cuenta el peso de las relaciones entre variables estructurales, se procedió a eliminar las dos dimensiones con menores correlaciones dentro de un proceso conocido como Backward Elimination (Wang, Wright, Brownlee, \& Buswell, 2016), calculando nuevamente los resultados del modelo cada vez que fue requerida la eliminación de una dimensión. En la solución, bajo la técnica PLS-SEM, se detectó también que las variables observables P29 y P30 tuvieron correlaciones cercanas a cero, de 0.022 y 0.021 , respectivamente. El modelo final se muestra en el Gráfico 3.

\section{Gráfica 3. Modelo final ajustado y resuelto}

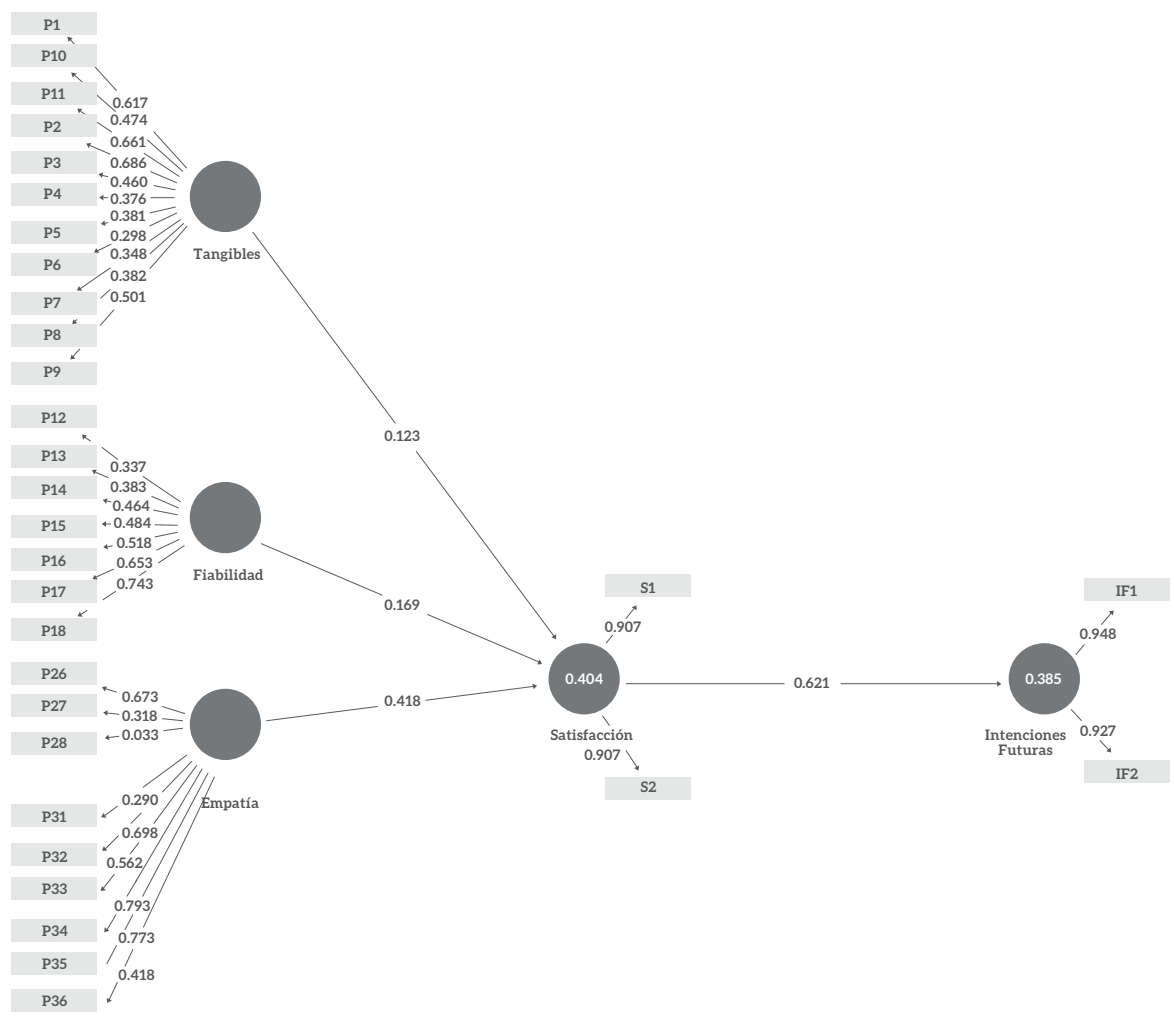

Fuente: Elaborado por los autores

Este modelo presenta correlaciones positivas entre las dimensiones y la satisfacción del turista nacional (Tabla 3). 
Tabla 3.

Resultados correlaciones positivas entre dimensiones y satisfacción

\begin{tabular}{l|c|c}
\hline Variables latentes & Intenciones Futuras & Satisfacción \\
\hline Empatía & & 0.418 \\
Fiabilidad & 0.621 & 0.169 \\
Satisfacción & & 0.123 \\
Tangibles & & \\
\hline
\end{tabular}

Fuente: Elaboración propia a partir de cuestionario aplicado a turistas nacionales.

Los valores de $\mathrm{R}^{2}$ no fueron afectados significativamente con respecto al modelo inicial propuesto. Con respecto a la validez del modelo, se obtuvieron valores adecuados para la Fiabilidad Compuesta y AVE superiores al valor recomendado de 0.5. El Alfa de Cronbach mínimo fue de 0.81 . Por otro lado, el factor de inflación de la varianza (VIF) fue inferior a 10, mostrando la no presencia de multicolinealidad en los resultados del modelo (Henseler, Ringle, \& Sinkovics, 2009)

De acuerdo con los resultados del modelo, una vez aplicada la simulación mediante la técnica Boostrap (basado en una muestra de tamaño igual a 1000), se concluyó que, de las 6 hipótesis planteadas, 4 fueron aceptadas (Tabla 4). Estos resultados indican que existen correlaciones significativas positivas entre la empatía, fiabilidad y los elementos tangibles respecto a la satisfacción, cuya variación es explicada por estas tres dimensiones en aproximadamente un 40\%. El modelo mostró que la empatía posee la mayor correlación positiva de 0.417 (media de la muestra) con respecto a la satisfacción, es decir, que un incremento en la puntuación de las variables que componen la empatía afectará directamente la satisfacción del usuario. En cuanto a la fiabilidad, esta posee una correlación positiva de 0.175 , de elementos tangibles de 0.130, e intenciones futuras de 0.624, siendo esta ultima la que mayor tiene correlación con la satisfacción.

Tabla 4.

Aceptación de las Hipótesis

\begin{tabular}{l|c}
\hline Hipótesis & Resultado \\
\hline $\begin{array}{l}\text { H1: Existe un efecto causal directo entre las variables tangibles y la } \\
\text { satisfacción del turista nacional. }\end{array}$ & Aceptado \\
\hline $\begin{array}{l}\text { H2 } 2_{0} \text { : Existe un efecto causal directo entre las variables de fiabilidad y la } \\
\text { satisfacción del turista nacional. }\end{array}$ & Aceptado \\
\hline $\begin{array}{l}\text { H3: Existe un efecto causal directo entre las variables de capacidad } \\
\text { de respuesta y la satisfacción del turista nacional. }\end{array}$ & Rechazado \\
\hline $\begin{array}{l}\text { H4: Existe un efecto causal directo entre las variables de seguridad } \\
\text { y la satisfacción del turista nacional. }\end{array}$ & Rechazado \\
\hline $\begin{array}{l}\text { H5: Existe un efecto causal directo entre las variables de empatía y la } \\
\text { satisfacción del turista nacional. }\end{array}$ & Aceptado \\
\hline $\begin{array}{l}\text { H5: H60: Existe un efecto causal directo entre las variables de satisfacción } \\
\text { e intenciones futuras del turista nacional. }\end{array}$ & Aceptado
\end{tabular}

Fuente: Elaboración propia de los autores. 


\section{CONCLUSIÓN}

El presente estudio tuvo como propósito valorar la calidad del servicio turístico de Cartagena de Indias a partir de los productos y servicios que ofrece el destino evaluados desde la perspectiva de los turistas nacionales, mediante la identificación de variables latentes y observables, el diseño de un instrumento, el planteamiento de seis hipótesis basadas en un modelo causal resuelto mediante la técnica de Mínimos Cuadrados Parciales, estableciendo las relaciones significativas entre atributos e identificando los elementos clave que afectan la satisfacción.

El modelo fue aplicado exitosamente dentro del contexto del servicio turístico de la ciudad, demostrando la alta relación entre calidad percibida, satisfacción e intenciones futuras de los visitantes nacionales de la ciudad de Cartagena de Indias, brindando solución al problema de investigación planteado. La aplicación del estudio condujo a la validación del modelo propuesto, sustentado teóricamente y empíricamente, con condiciones generales que facilitarían la evaluación del servicio turístico de Cartagena, enfocado en las cinco dimensiones establecidas en la fase de presentación del proyecto.

Luego de la aplicación del modelo causal se encontró que las intenciones futuras son determinantes para la satisfacción del turista nacional respecto a Cartagena como destino. Dimensiones como empatía, capacidad de respuesta y tangibilidad también tienen una alta correlación con la satisfacción. Por lo anterior, se concluye que de las seis hipótesis presentadas se aceptaron las cuatro enunciadas (H1, H3, H5 y H6). Es de anotar que los resultados de este proyecto permitieron darle aplicación a la técnica de Mínimos Cuadrados Parciales -PLS- para la valoración del servicio de Cartagena de Indias como destino turístico, limitado en la aplicación del instrumento en la temporada de fin de año entre diciembre de 2017 y enero de 2018, y adecuado al contexto de la ciudad como destino turístico.

El beneficiario directo de este proyecto es el sector turístico de la ciudad, especialmente la Corporación Turismo Cartagena de Indias, como representante del sistema de gestión del Centro Histórico y del destino como tal, puesto que luego de identificados los factores que influencian la satisfacción del turista, las variables que afectan la calidad del servicio y las recomendaciones que a continuación se presentan, el destino podrá tomar correctivos que le permitan mejorar la competitividad y productividad de la ciudad, puesto que ofreciendo un servicio con una mejor calidad para los usuarios, se contribuirá a la sostenibilidad del destino.

Para investigaciones futuras se recomienda que sea incluido el perfil sociodemográfico de los encuestados en el modelo. Así mismo, que en el levantamiento de información se incluyan turistas internacionales y aumentar el periodo de tiempo en que se lleva a cabo el mismo, teniendo en cuenta las políticas del Gobierno Nacional frente al aumento de las divisas en el país con el fin de que el turismo sea una de las principales fuentes de desarrollo económico. 


\section{REFERENCIAS BIBLIOGRAFICAS}

Cassel, C., Hackl, P., \& Westlund, A. H. (1999). Robustness of partial least- squares method for estimating latent variable quality structures. Journal of Applied Statistics, 26(4), 435-446. https://doi.org/10.1080/02664769922322

Centro de Información Turística de Colombia. (2018). Estadísticas Nacionales - Turísmo Receptor. Retrieved from http://citur.linktic.com/estadisticas/df_ cruceros/barcos/8

Centro de Pensamiento Turístico - Colombia. (2018). Índice de Competitividad Turística Regional de Colombia - ICTRC. Bogotá D. C.

Chin, W. W., \& Newsted, P. R. (1999). Structural Equation Modeling Analysis with Small Samples Using Partial Least Square. In Statistics Strategies For Small Sample Research (pp. 307-341). New York: SAGE Publications.

Cronin, J. J., \& Taylor, S. A. (1992). Measuring Quality: A Reexamination and. Journal of Marketing, 56(3), 55-68.

Crosby, P. B. (1992). The eternally successful organization : the art of corporate wellness. New York: First Mentor Priniting.

F. Hair Jr, J., Sarstedt, M., Hopkins, L., \& G. Kuppelwieser, V. (2014). Partial least squares structural equation modeling (PLS-SEM). European Business Review, 26(2), 106-121. https://doi.org/10.1108/EBR-10-2013-0128

Garvin, D. (1984). What Does "Product Quality" Really Mean? MIT Sloan Management Review, 26(1). Retrieved from https://sloanreview.mit. edu/article/what-does-product-quality-really-mean/

Garvin, D. (1987). Competing on the eight dimensions of quality. Harvard Business Review, 65(6), 101-109.

Gronroos, C. (1982). Strategic Management and Marketing in the Service Sector (Paperback). Helsingfors.

Henseler, J., Ringle, C. M., \& Sinkovics, R. R. (2009). Advances in International Marketing. Bingley: Emerald Group Publishing.

Holbrook, M. (2002). Intrduction to consumer value. In M. Holbrook (Ed.), Consumer Value: A framework for analysis and research (pp. 1-28). London: Routledge.

Monecke, A., \& Leisch, F. (2012). semPLS: Structural Equation Modeling Using Partial Least Squares. Journal of Statistical Software, 48(3), 1-32. 
Redacción Economía. (2019). 2018 fue el mejor año para el turismo en Colombia. El Espectador, p. 1. Retrieved from https://www.elespectador.com/ economia/2018-fue-el-mejor-ano-para-el-turismo-en-colombiaarticulo840013

Vergara-Schmalbach, J. C., \& Quesada, V. M. (2013). Valoración de la Calidad del Servicio Mediante Modelos de Ecuaciones Estructurales (No. 1). Cartagena de Indias: Universidad de Cartagena.

Vergara-Schmalbach, J. C., Quesada, V. M., \& Blanco, I. (2012). Factores clave para la valoración de la calidad del servicio y satisfaccion del cliente: Modelos causales, desarrollo y evolución. Revista Virtual Universidad Católica Del Norte, 35(1), 380-400.

Wang, M., Wright, J., Brownlee, A., \& Buswell, R. (2016). A comparison of approaches to stepwise regression on variables sensitivities in building simulation and analysis. Energy and Buildings, 127(1), 313-326. https://doi. org/10.1016/j.enbuild.2016.05.065

Zeithaml, V. A. (1981). How Consumer Evaluation Processes Differ Between Goods and Service. Marketing of Service, 9(1), 186-190.

Zeithaml, V. A., Berry, L. L., \& Parasuraman, A. (1988). Communication and Control Processes in the Delivery of Service Quality. Journal of Marketing, 52(2), 35. https://doi.org/10.2307/1251263

Zeithaml, V. A., Parasuraman, L. L., \& Berry, L. L. (1990). Delivering Quality Service: Balancing Customer Perceptions and Expectations. New York: Simon and Schuster. 